\title{
Motivations à mettre en lumière la culture muséale des enfants. Un enjeu pour la valorisation de l'éducation non formelle
}

\author{
Reasons to highlight children's museum culture: a stake in the \\ enhancement of non-formal education \\ Motivaciones para poner en relieve la cultura museística de los \\ niños: un reto para la valorización de la educación no formal
}

\section{Thérèse Martin}

Volume 43, numéro 1, printemps 2015

Vingt ans de recherche en éducation muséale

URI : https://id.erudit.org/iderudit/1030181ar

DOI : https://doi.org/10.7202/1030181ar

Aller au sommaire du numéro

Éditeur(s)

Association canadienne d'éducation de langue française

ISSN

1916-8659 (numérique)

Découvrir la revue

Citer cet article

Martin, T. (2015). Motivations à mettre en lumière la culture muséale des enfants. Un enjeu pour la valorisation de l'éducation non formelle. Éducation et francophonie, 43(1), 63-79. https://doi.org/10.7202/1030181ar

\section{Résumé de l'article}

Nos travaux de recherche en muséologie s'intéressent à la réception du " public-enfants », en particulier dans les musées de sciences. Pour situer le cadre de notre recherche, nous proposons de mieux cerner la bipolarité du musée dont la mission est souvent partagée entre éducation, pédagogie, apprentissage, d'une part, et loisir, délectation, désir de se cultiver, d'autre part. Face notamment à l'influence de la pédagogie scolaire dans les musées en France, il nous a paru nécessaire d'étudier en quoi le musée peut être un " outil » de culture, en revenant sur les fondements des premiers musées pour enfants et sur les pédagogies actives des musées de sciences dans une perspective d'acculturation. Notre recherche porte sur la compréhension des manières dont les enfants s'approprient le média-exposition dans un cadre de loisirs (en famille). Ce sont les " fabrications culturelles " des enfants qui ont été étudiées lors de l'expérience de visite considérée sur l'ensemble du parcours et selon l'ensemble des dimensions qui la traversent. Des figures d'enfants-interprètes alors dégagées de l'analyse des visites des enfants rendent compte de la richesse des modes d'appropriation de l'exposition et peuvent être source d'inspiration pour les professionnels des musées et les médiateurs d'exposition (enseignants, guides).
Tous droits réservés ( Association canadienne d'éducation de langue française, 2015 cecument est protégé par la loi sur le droit d’auteur. L'utilisation des services d’Érudit (y compris la reproduction) est assujettie à sa politique d'utilisation que vous pouvez consulter en ligne. 


\section{Motivations à mettre en lumière la culture muséale des enfants}

Un enjeu pour la valorisation
de l'éducation non formelle

Thérèse MARTIN

Université de Bordeaux-Montaigne, Bordeaux - France

\section{RÉSUMÉ}

Nos travaux de recherche en muséologie s'intéressent à la réception du «publicenfants», en particulier dans les musées de sciences. Pour situer le cadre de notre recherche, nous proposons de mieux cerner la bipolarité du musée dont la mission est souvent partagée entre éducation, pédagogie, apprentissage, d'une part, et loisir, délectation, désir de se cultiver, d'autre part. Face notamment à l'influence de la pédagogie scolaire dans les musées en France, il nous a paru nécessaire d'étudier en quoi le musée peut être un «outil» de culture, en revenant sur les fondements des premiers musées pour enfants et sur les pédagogies actives des musées de sciences dans une perspective d'acculturation. Notre recherche porte sur la compréhension des manières dont les enfants s'approprient le média-exposition dans un cadre de loisirs (en famille). Ce sont les «fabrications culturelles» des enfants qui ont été étudiées lors de l'expérience de visite considérée sur l'ensemble du parcours et selon l'ensemble des dimensions qui la traversent. Des figures d'enfants-interprètes alors dégagées de l'analyse des visites des enfants rendent compte de la richesse des 
modes d'appropriation de l'exposition et peuvent être source d'inspiration pour les professionnels des musées et les médiateurs d'exposition (enseignants, guides).

\title{
ABSTRACT
}

\section{Reasons to highlight children's museum culture: a stake in the enhancement of non-formal education}

Thérèse MARTIN

University of Bordeaux Montaigne, France

Our museology research is about how museums, in particular science museums, host a young public. To situate the context of our research, we define the bipolarity of the museum, whose mission is often shared between education, teaching and learning on one hand, and leisure, pleasure, and the appreciation of culture on the other. We will show that because of the influence of school pedagogy on French museums, we felt it necessary to study how the museum could be a cultural "tool", going back to the foundations of the first children's museums and the active pedagogy of science museums through a perspective of acculturation. Our research focuses on understanding the ways in which children take ownership of the exhibition media in a recreational context (with the family). We studied their "cultural fabrications" during the visiting experience, including each stage of the visit and all of its dimensions. Figures of child-interpreters drawn from the analysis of children's visits reflect the wealth of ways in which children experience their visits, and can be sources of inspiration for museum professionals and exhibition mediators (teachers, guides).

\section{RESUMEN}

\section{Motivaciones para poner en relieve la cultura museística de los niños: un reto para la valorización de la educación no formal}

\author{
Thérèse MARTIN \\ Universidad de Bordeaux Montaigne, Francia
}

Nuestros trabajos de investigación en museología se interesan en la recepción del público-niño, particularmente en los museos de ciencias. Con el fin de apreciar el marco de nuestra investigación se propone acotar esta bipolaridad del mueso cuya misión con frecuencia se comparte entre educación, pedagogía, aprendizaje, por una parte, y el esparcimiento, el deleite, el deseo de cultivarse por otra parte. Mostraremos que sobre todo frente a la influencia de la pedagogía escolar en los muesos en Francia, nos ha parecido necesario estudia de qué manera el museo puede ser un «instrumento» de cultura, retornando a las bases de los primeros 
muesos para niños y a las pedagogías activas de los museos de ciencia en una perspectiva de aculturación. Nuestra investigación aborda la comprensión de las formas en que los niños se apropian el medio-exposición en el marco de un esparcimiento (en familia). Lo que estudiamos son sus «fabricaciones culturales» durante la visita considerada como un conjunto del recorrido y de acuerdo al conjunto de dimensiones por la que pasan. Gracias al análisis de las visitas se extraen las figuras del niño-interprete que permite exponer la riqueza de los modos de apropiación de la visita y pueden servir de fuentes de inspiración para los profesionales de los museos y de los intermediarios de la exposición (maestros, guías)

\section{Introduction}

Les musées en tant que lieux d'éducation non formelle contribuent à la diffusion de formes de savoirs et de culture. Les institutions muséales ont été beaucoup étudiées sous l'angle de l'éducation, alors que la dimension culturelle est restée peu valorisée. Face à l'abondance des recherches sur l'apprentissage, au constat de l'influence de l'école dans les musées en France et de son effet de "scolarisation» de la visite, il a été utile de rechercher les raisons pour lesquelles le musée en tant qu' «outil» de culture est resté dans l'ombre.

Ce sont autant de motifs qui ont justifié l'orientation de notre recherche consistant à explorer où se «niche» en quelque sorte la dimension culturelle du musée. À travers l'historique du musée et sa mission, la distinction entre institutions muséales et institutions éducatives, les caractéristiques de l'éducation non formelle et la tentative de définition de la situation d'éducation non formelle dans le cas particulier des visiteurs-enfants, mais aussi à travers la communication par l'exposition et les formes pédagogiques à l'œuvre dans les musées de sciences, nous tentons d'extraire ce qui laisse entrevoir la visée culturelle du musée afin de mieux cerner celle-ci. Un retour aux fondements à l'origine du musée pour les enfants sera le point de départ du cadre fixé pour étudier ce qui se joue dans l'espace de médiation de l'exposition, à savoir ce que "font» les enfants avec la communication de l'exposition, en d'autres termes, leurs manières de s'approprier l'exposition dans le cadre des loisirs. Cette recherche en sciences de l'information et de la communication a pour ambition de mettre en lumière la culture muséale des enfants - une façon de valoriser les enjeux de l'éducation non formelle.

Cet article se réfère principalement au contexte muséologique en France, mais s'inspire de travaux de recherche du Québec ou des États-Unis, pour leurs apports en lien avec notre préoccupation de recherche. 


\section{Le musée, un contexte d'éducation non formelle}

\section{Une volonté éducative du musée}

Un bref historique permet de signaler que le musée, créé en 1792 en France, par décret de la Convention, fut - selon son acception moderne - conçu d'emblée comme un lieu de formation, soit une institution conservatoire ouverte à tous pour l'étude et la délectation. Une volonté éducative s'est clairement affirmée avec les musées de la fin du $\mathrm{XX}^{\mathrm{e}}$ siècle, en même temps qu'une vocation communicationnelle des expositions, selon Poulot (2005). Cette situation prévaut après une longue période où le musée est axé principalement sur la fonction de conservation. Toutefois, le jeune "public» ne fait pas l'objet d'une prise en compte particulière.

Concernant les fonctions muséales, Meunier (2012, p. 104-105) en rappelle l'évolution selon la mission du musée. À l'époque de Rivière, premier directeur du Conseil international des musées (ICOM) ${ }^{1}$, cette mission repose sur les fonctions de recherche, de conservation et de présentation. Dans la définition des musées adoptée par l'ICOM en 1951, il est question de «conserver, étudier, mettre en valeur [...] et essentiellement exposer pour la délectation et l'éducation du public un ensemble d'éléments de valeur culturelle». La définition de 1974, que l'on retrouve en 2001 dans les statuts de l'ICOM², précise le rôle du musée: conserver, communiquer et notamment exposer à des fins d'études et de délectation. Nous pouvons remarquer que, depuis 1951, la dimension plaisir est présente et associée à celle d'éducation et d'études, sachant que la délectation est définie comme un «plaisir sensible ou d'ordre intellectuel que l'on savoure pleinement», selon le Trésor de la langue française.

\section{L'éducation non formelle, distincte de l'éducation formelle}

Les musées contribuent à la diffusion de formes diverses de savoir et de culture; c'est en cela qu'ils se distinguent des institutions éducatives. Pour Jacobi et Coppey (1995), «les modes d'appropriation de la culture ou du savoir qu'ils proposent diffèrent nettement de la sphère scolaire» (p. 11). C'est ainsi que les musées sont rangés dans le secteur de l'éducation non formelle ${ }^{3}$, par opposition aux établissements scolaires (écoles, collèges...) qui «dispensent, à des groupes d'élèves d'âge homogène, un enseignement académique (dit formel), défini au préalable par des instructions

1. ICOM : Organisation internationale non gouvernementale des musées et des professionnels de musée ayant, entre autres, comme principaux objectifs de «faire mieux connaître et comprendre la nature, les fonctions et le rôle des musées au service de la société et de son développement et faire progresser et diffuser la connaissance dans les domaines de la muséologie et des autres disciplines concernées par la gestion et les activités du musée». Statuts de l'ICOM, article 1, paragraphe 1. Lettre du Comité national français, numéro spécial, code de déontologie, n²22, mai 1993.

2. "Le musée est une institution permanente, sans but lucratif, au service de la société et de son développement, ouverte au public et qui fait des recherches concernant les témoins matériels de l'homme et de son environnement, acquiert ceux-là, les conserve, les communique et notamment les expose à des fins d'études, d'éducation et de délectation» (Statuts de l'ICOM, article 2, paragraphe 1).

3. À propos de "informal education», la traduction littérale "éducation informelle» est remplacée par le concept d'éducation non formelle (opposée à formelle). Pour D. Jacobi et B. Schiele, le terme d' «éducation informelle» n'est pas approprié, car il n'évite pas le risque que lui soient attribuées les caractéristiques «d'absence de structuration, d'organisation et, institutionnellement, l'absence de réelle consistance... » (Jacobi et Schiele, 1990). 
officielles, des règles et des usages fortement codifiés " (Jacobi et Coppey, 1995, p. 11). Si les institutions muséales se différencient des institutions éducatives, cela conduit à explorer la manière dont l'éducation formelle se distingue de l'éducation non formelle. Selon Jacobi et Schiele (1990), l'éducation non formelle consiste à choisir ses méthodes et ses objectifs en dehors de contraintes, telles que des instructions officielles. Pour les musées, l'objectif de l'éducation non formelle est de choisir ses thèmes librement (en rapport avec l'actualité, par exemple), en veillant à répondre aux questions qui préoccupent les personnes à qui elles s'adressent. Le public est non captif. Pour se situer du côté du visiteur de l'exposition de sciences, une exposition qui relève de l'éducation non formelle offre au visiteur «la possibilité de s'arrêter plus longtemps sur certains éléments, de sauter d'autres éléments et de se construire son propre parcours» (Davallon, 1998, p. 263). C'est au gré du visiteur que se réalise le parcours de visite.

L'institution muséale en tant que contexte d'éducation non formelle n'est pas soumise aux contraintes de contenus liées aux programmes scolaires, comme c'est le cas des institutions éducatives. Pour les visiteurs, l'exposition est propice à une exploration à leur gré, tout en construisant leur propre parcours.

\section{L'éducation non formelle et la situation de visite dans le cas de visiteurs-enfants}

Des précisions sont apportées par Jacobi et Coppey (1995) quant au contexte de l'éducation non formelle, en direction d'un public d'adultes:

La visite du musée est une activité volontaire, prise sur son temps de loisir, de type intermittent, sans programme préétabli et sans système de sanction et de récompense susceptible de lui conférer une énergie motivationnelle. On peut aussi ajouter qu'il s'agit d'une activité sans médiateur où la détente, la délectation, ou parfois même le jeu, ne sont jamais très loin du désir d'apprendre et de se cultiver (p. 12).

Une proximité avec la détente (un parcours choisi par le visiteur, sans contraintes), avec la délectation, voire avec le jeu (forme interactive fréquente notamment dans les musées de sciences), est soulignée par ces auteurs. On peut en déduire qu'apprendre et se cultiver peut passer par le loisir, le plaisir intellectuel ou sensible et le jeu. Les auteurs ajoutent que la situation de visite pour des enfants est plus difficile à situer dans un cadre d'éducation non formelle, par le fait que les enfants ont, en général, besoin d'un médiateur (leur accompagnateur) et que ces activités muséales peuvent être plus difficiles à distinguer des activités d'éducation formelle, si les enfants ont l'habitude de visiter les musées avec l'école. Une difficulté est soulevée ici, celle de définir les situations d'éducation non formelle en tant que telles. C'est en opposition avec le fonctionnement de l'institution scolaire (autrement formulé en termes de bipolarité) ou avec l'activité des élèves que l'éducation non formelle est située, selon Jacobi et Coppey (1995), et non en termes d'ouverture culturelle. 


\section{En quête de la dimension culturelle des musées}

\section{Vers une acculturation}

Pour qualifier les apports culturels possibles d'une visite dans un lieu culturel, pour un visiteur qui n'est pas un habitué, le terme «acculturation» est privilégié, puisque sa définition évoque une approche d'une culture nouvelle par «une multiplicité de microprocessus, d'invention, d'imitation, d'apprentissage ou d'adaptation» (Traité de sociologie, 1968, p. 318, cité dans Le Trésor de la langue française informatisé du CNRS). Le terme "acculturation» est utilisé par Jacobi et Coppey (1995) lorsqu'ils s'interrogent sur le moyen de passer d'un mode éducatif à un mode culturel, qu'il s'agisse de services, d'actions ou d'impacts de l'exposition. C'est en termes de "capacité ${ }^{4}$ » à pouvoir utiliser souplement des contenus des musées pour les intégrer avec l'existant, qu'est abordée cette notion (p. 18). Cependant, cela suscite la question de savoir de quelle manière, en dehors de ces stratégies cognitives, il est possible d'identifier et de caractériser cette acculturation au cours de la visite.

\section{La culture, un mouvement inverse à la communication pédagogique}

Selon Davallon (2005), "l'usage du terme “éducation” souvent utilisé reste ambigu, car il se situe à la limite de la pédagogie et de la culture» (p. 258). C'est en établissant une distinction entre la communication par l'exposition et la communication pédagogique que Davallon fait cette remarque. Le modèle éducatif de la relation public/institution, tout en voulant rendre accessible à tous le savoir divulgué par les expositions de sciences, du fait de son inscription dans la vulgarisation scientifique, a pour effet d'occulter la dimension culturelle du musée. Cette situation s'explique, selon Davallon (2005), par la visée unidimensionnelle d'acquisition de connaissances ou d'apprentissage du modèle pédagogique, même si celui-ci s'appuie sur la dimension sensible ou affective (p. 258). Il est opportun de rappeler ici, au cours des trente dernières années, après avoir été centrées sur la simple acquisition de connaissances, que «les études [sur les visiteurs au niveau international] vont s'intéresser à tout ce qui peut jouer sur l'apprentissage: phénomènes cognitifs, affectifs, sociaux" (en fonction des bases de données anglophones) (Davallon, Gottesdiener et Vilatte, 2006, p. 162). Par ailleurs, les évaluations introduites par Screven (1990) soutiennent que l'exposition doit être conçue pour le visiteur en s'appuyant sur des objectifs prédéterminés - les d'apprentissages acquis étant comparés à ces objectifs ${ }^{5}$ - ont renforcé la conviction qu'un musée est un milieu éducatif dont «la mission est de transmettre des connaissances et de permettre des apprentissages ", comme le rappelle Schiele (2002, p. 123).

4. Cette capacité à utiliser souplement l'exposition est la marque d'une acculturation achevée, pas tant aux contenus des musées (tant ces derniers sont riches et multiples) qu'au mode d'emploi de ces derniers par un visiteur autonome, libre de ses choix et capable de les intégrer dans un système cohérent de valeurs (Jacobi et Coppey, 1995, p. 18).

5. "La démarche d'évaluation nécessite de clarifier préalablement les objectifs cognitifs et affectifs de l'exposition, d'énoncer les objectifs comportementaux pratiques et de mesurer l'atteinte de ces objectifs. Les objectifs doivent être mesurables», (Schiele, 2002: 123) 
Au Québec, Meunier (2008), observe que les actions d'éducation muséale cherchent à se démarquer de l'école et que l'offre d'activités «n'est que rarement originale, novatrice et renouvelée» (p. 105), même si elle s'avère intéressante ${ }^{6}$.

Plutôt qu'une visée unidimensionnelle d'apprentissage, c'est un mouvement inverse qui est préconisé par Davallon (2005), à savoir «un dynamisme, une densité et un foisonnement dus au branchement de logiques et d'éléments hétérogènes» (p. 259) qui caractérise la culture. On pourrait imaginer que les manières d'approcher les formes de savoirs du musée répondent à ce mouvement inversé, grâce à un milieu riche à explorer autrement qu'avec la préoccupation d'évaluation de l'acquisition d'apprentissages. Face à un modèle pédagogique parfois trop prégnant, nous préférons nous tourner vers la situation de médiation (ou situation de communication par l'exposition) afin d'étudier les réactions des visiteurs et privilégier cette dynamique favorisant la culture.

\section{Les perspectives d'acculturation à travers les pédagogies actives des musées de sciences}

En ce qui concerne plus spécifiquement les musées de sciences, l'ouvrage de Eidelman et Van Praët (2000) permet de mieux saisir l'origine de la dimension éducative dans les musées, à propos de l'histoire de la muséologie. Selon ces deux auteurs, les musées de sciences ont hérité de la réforme des contenus de l'enseignement scientifique, de la réflexion sur les relations pédagogiques (pédagogie active) qui marque la décennie 1960-70 des sciences de l'éducation, avec Giordan ${ }^{7}$ et de la didactique des disciplines (Hulin et Guichard, 1990) ${ }^{8}$. Ainsi au Palais de la Découverte, on montre «la science en train de se faire» afin de «de populariser les avancées d'une recherche scientifique basée sur l'expérience», grâce à «une "théâtralisation" d'un parti pris épistémologique majoritaire au sein de la communauté scientifique à une époque donnée» (Eidelman et Van Praët, 2000, p. 15). Les auteurs procèdent à une distinction entre la muséologie de l'implication et celle de l'émotion $^{9}$. On note ici la place accordée à l'émotion pour sensibiliser, toucher le public. Il nous semble important que cette dimension sensible soit prise en considération, car elle est constitutive de l'expérience de visite.

L'Exploratorium de San Francisco, créé en 1969 par le physicien Oppenheimer, a révolutionné la pratique de la muséologie scientifique en voulant faire du musée un lieu de médiation des connaissances. Le visiteur est situé au centre du dispositif, et sa participation active est sollicitée par un nombre important de manipulations. Delacôte (1996), qui compte parmi les fondateurs du musée scientifique de la Cité

6. «On se trouve plutôt souvent face à une répétition d'approches qui ont fait plus ou moins bien leurs preuves, qui satisfont à peu près les visiteurs et les participants et qui atteignent à peu près les intentions éducatives poursuivies à l'intention des élèves-visiteurs» (Meunier, 2008, p. 105).

7. Voir la bibliographie de A. Giordan à http://www.andregiordan.com/biblio.html

8. Ce qui explique, par ailleurs, que les musées de sciences ont fréquemment été à l'origine des transformations de la pédagogie muséale dans la mesure où ils ont été «la matrice» (Eidelman et Van Praët, 2000, p. 4).

9. On peut distinguer la "muséologie de l'implication» sous la forme d'« invitation à toucher » par l'entremise de dispositifs à boutons-poussoirs et la muséologie de l'émotion par les expériences spectaculaires réalisées en direct par le personnel de l'établissement (Eidelman, 1998, cité dans Eidelman et Van Praët, 2000, p. 5). 
des sciences et de l'industrie de la Villette, à Paris, a lui-même été directeur de l'Exploratorium. Pour contribuer à améliorer l'éducation et donner sa chance à chacun, il a retenu plusieurs principes qui ont régi cette institution muséale: une maîtrise des processus cognitifs et sociocognitifs d'apprentissage grâce à une meilleure connaissance de ces processus (Delacôte, 1996, p. 257) et une conception d'environnements d'apprentissage interactifs, qui doivent être stimulants, engageants, cognitivement efficaces et économiquement raisonnables (Delacôte, 1996, p. 259). Ces outils qui doivent être conçus pour un usage collectif doivent également susciter la curiosité ${ }^{10}$. C'est à ces conditions que «[L]'apprentissage scientifique [...] est considéré comme un processus d'acculturation au monde des pratiques et des discours scientifiques, offrant l'accès à un savoir expérimental, symbolique et négocié socialement, qui doit donc être étudié et compris dans un contexte» (Delacôte, 1996, p. 258). De nos jours, ces fondements sont encore de mise à l'Exploratorium. Avec la mission de renouveler l'accès aux savoirs grâce à des environnements d'apprentissage innovants, ce musée se présente comme le chef de file de l'éducation non formelle pour susciter la curiosité et inspirer la créativité aux visiteurs de tout âge grâce à la participation de nombreux chercheurs comme membres du personnel.

Les expositions de sciences retenues pour nos expérimentations sont des expositions temporaires avec une trame narrative et dont la médiation est très présente. Les scènes de l'exposition Le festin des dinosaures touchent la sensibilité des enfants et les interrogent sur la vie des dinosaures. La mise en scène de l'exposition Ombres et lumière associe habilement art et sciences. Ces expositions sont propices à éveiller la curiosité des visiteurs-enfants. Elles sont en mesure de donner lieu à des situations de communication riches et variées.

\section{Le choix d'un cadre pour remédier à la tension entre les visées éducative et culturelle}

Selon les didacticiens des sciences, tels que Guichard et Martinand (2000), la vulgarisation scientifique des musées de sciences a une visée culturelle dans la mesure où les usagers utilisent les médias scientifiques sur leur temps de loisirs pour se distraire, non pour s'ennuyer ou pour étudier (p. 38). Mais aussi, pour ces chercheurs, la mission éducative est liée au souci d'éducation du citoyen en face d'un monde en changement, pour faire découvrir ses transformations. Ainsi, ils proposent de surmonter la tension entre la visée culturelle et la mission éducative par le choix entre le cadre de loisirs et le cadre scolaire de la visite. Pour notre recherche, c'est le cadre de loisirs (en famille) qui a été retenu pour étudier les réactions des enfants au moment de leur visite de l'exposition.

10. Selon Delacôte (1996), la création de ces outils d'exploration d'un phénomène naturel requiert beaucoup de talent. «Il faut savoir surprendre, donner à observer, ne pas craindre la complexité, mais en même temps permettre la compréhension; [la visée est] de créer par l'étonnement une dynamique cognitive» (p. 77). 


\section{Valoriser le musée comme outil de culture en contexte d'éducation non formelle}

\section{Un retour aux sources: la place accordée à l'expérience dans les musées pour enfants}

L'historique du musée pour les enfants montre l'intérêt de l'institution sur le temps de loisirs (en complément de l'école) et, surtout, la nécessité d'une adaptation propre aux besoins particuliers des enfants, si l'on en croit Dewey (2006) et ses principes fondateurs de philosophe et pédagogue.

Gurian (2006) trace un résumé de l'histoire des children's museums (et des salles apparentées destinées aux enfants dans les grands musées). Notamment, elle mentionne les objectifs du premier musée pour enfants au monde, créé à Brooklyn en 1899, qui étaient les suivants:

Proposer un lieu attrayant pour les enfants, qui les incite à affiner leurs goûts et à élargir leurs centres d'intérêt; créer un centre pédagogique intéressant qui apporte une assistance quotidienne aux élèves et aux professeurs, en rapport avec leur travail scolaire et propose de nouveaux sujets de réflexion pour occuper leur temps libre. (Gallup, 1907, cité par Gurian, 2006, p. 8)

Ces objectifs confèrent au musée une double orientation, à la fois d'éducation pour apporter une complémentarité à l'école et de loisir pour développer les centres d'intérêt des enfants, avec des méthodes favorisant la curiosité. Dès la création des musées pour enfants, les intentions pédagogiques qui les guident sont axées sur l'expérience des jeunes visiteurs, dans un contexte attrayant pour éveiller leurs goûts et enrichir leurs découvertes. Elles s'appuient sur les idées philosophiques énoncées par Dewey (2006), en particulier sur la théorie axée sur l'expérience tactile (handson), qui insiste sur la nécessité pour les jeunes enfants de pouvoir expérimenter, toucher, observer et examiner du matériel tangible afin d'améliorer leur éducation. Selon cette théorie philosophique, les enfants ne sont pas de petits adultes; ils présentent un développement différent, qui exige des méthodes pédagogiques fondées sur leurs besoins particuliers. À ce sujet, Gurian (2006) souligne que Dewey (1969; cité dans Peters, 1977, p. 60) a écrit qu'«il doit y avoir plus de matériel concret, plus de "trucs", plus d'appareils et plus de possibilités de faire des choses ${ }^{11} \ldots$.. (p. 8).

\section{La mise en évidence des "fabrications culturelles " des enfants}

Malgré les intentions des musées, à l'origine pour favoriser la curiosité chez les enfants en les incitant à affiner leur goût par une ouverture sur différents centres d'intérêt, la question est restée entière sur leurs manières dont ils s'approchent de l'exposition et se l'approprient. C'est encore plus vrai lorsqu'il s'agit de considérer

11. À titre d'exemple, Anne Billings Gallup, premier conservateur du Musée pour les enfants de Brooklyn, raconte qu'elle ouvrait les vitrines de manière à ce que les enfants puissent toucher aux objets (Zervos, 2002, cité dans Gurian, 2006). 
l'expérience de visite dans sa globalité, c'est-à-dire sur l'ensemble des dimensions qui la traversent et sur la totalité du parcours de visite. C'est ainsi que, pour comprendre l'appropriation de l'exposition par un jeune public, nous avons fait le choix d'étudier ce que «fabriquent» les visiteurs-enfants ou ce qu'ils «bricolent», à la lumière de la "culture ordinaire», telle que définie par de Certeau (1974). Ce qui revient à étudier ce qui se joue dans cet espace de médiation, entre les visiteurs et l'exposition. Pour ce philosophe, la «culture ordinaire» est faite de pratiques sociales qui ont « une signification pour celui qui les effectue». À l'instar de l'auteur, il convient de prendre en compte dans la culture «l'activité propre de l'individu, l'appropriation des langages et des valeurs, la transformation de son identité» (p. xxxv).

\section{Les figures d'enfants-interprètes: le reflet d'une culture muséale en construction}

La visée d'acculturation des visiteurs-enfants (et par la même l'occasion de faire du musée un «outil» de culture) se manifeste à travers leurs «fabrications culturelles» qui rendent compte de leur interprétation. Celles-ci s'inscrivent dans l'expérience de visite en référence au philosophe Dewey (2006) - considérée sur la totalité du parcours et sur l'ensemble des dimensions qui la traversent - et elles sont arrimées au discours de l'exposition par l'intermédiaire des espaces discursifs de l'exposition (Silverstone, 1998). Les paroles des visiteurs-enfants ont été recueillies lors de la visite en famille et lors de la visite «guidée» par les enfants.

\section{La méthodologie mise en œuvre: un dispositif d'enfant-médiateur}

Les expérimentations ont eu lieu à Paris dans deux expositions temporaires à thèmes: l'exposition Le festin des dinosaures au Palais de la Découverte et l'exposition Ombres et lumière à la Cité des enfants de la Cité des sciences et de l'industrie, au parc de la Villette. Dix-neuf enfants ont fait l'objet d'observations et ont été des guides pour le chercheur. Parmi les méthodes existantes, l'entretien itinérant donne accès à l'interprétation du visiteur (adulte) en lui demandant « de décrire au fur et à mesure ce qu'il vit, ce qu'il voit, pense, imagine, ressent" (Dufresne-Tassé et Lefebvre, 1996, p. 14). Cependant, il nous a paru difficile de demander à un enfant à la fois de vivre en direct l'expérience et de devoir prendre un minimum de recul pour mettre des mots sur ce qu'il vit en guise d'explicitation. Il a été nécessaire par conséquent d'élaborer un dispositif méthodologique communicationnel, «la mise en situation de rôle de guide», pour rendre compte des «fabrications culturelles» des enfants au cours de leur expérience de visite. Ce dispositif constitue un «laboratoire» d'observations de l'interprétation en train de se faire. Il révèle les habiletés des enfants à remplir le rôle de "médiateur" et à être acteurs, auteurs de leur visite guidée. Ce dispositif présente l'intérêt de mettre en évidence les relations établies par les visiteurs-enfants entre les objets, le lieu, les personnages et l'institution. 


\section{L'émergence de cadres d'interprétation des enfants}

Quant à l'analyse des «visites guidées» des enfants et de leurs récits, elle est conduite en trois phases, selon une trame d'analyse détaillée. Celle-ci s'appuie sur le parcours effectué, la médiation par le récit (les relations établies avec les objets, le lieu, l'institution, le savoir), leurs manières de construire un monde (selon les opérations de description effectuées ${ }^{12}$ ), l'évocation de leurs propres références, leurs manières de «guider» leur visiteur (soit les postures, les gestes, les descriptions), les formes de la «visite guidée» (intentions du "guide», prise en mains de la situation de guide selon le choix des objets, du parcours, utilisation des cartels ou non...), les expériences vécues en fonction des espaces discursifs de l'exposition, la circulation de la médiation à partir de la combinaison de ces expériences (visite en famille, visite avec le chercheur).

La première phase d'analyse consiste à dégager, de manière empirique, les cadres d'interprétation mis en œuvre par les enfants. À cette fin, l'expérience de visite est alors étudiée sur l'ensemble des dimensions qui la traversent, plus précisément selon les façons dont les enfants s'approprient les espaces discursifs de l'exposition (l'espace de la rhétorique, du jeu, de la performance) en tant que lieux de construction de sens (Silverstone, 1998) et l'espace de l'esthétique plus spécifique aux expositions dont la médiation est sensible ${ }^{13}$.

Pour une illustration de la démarche d'analyse (cadres d'interprétation et figures d'enfants-interprètes), des exemples d'analyse de "visites guidées» sont donnés dans les annexes de Martin (2011).

\section{Les figures d'enfants-interprètes, un éclairage sur l'interprétation des enfants}

Selon une approche longitudinale, le deuxième temps d'analyse étudie l'expérience de visite des enfants "guides" sur l'ensemble du parcours. En fonction de composantes que nous avons déterminées, il s'agit d'identifier les différentes configurations possibles de la démarche interprétative des enfants. Ces composantes sont principalement l'intention de visite, le mode d'appropriation, la prise en compte (ou non) du propos de l'exposition donnant des indications sur leur intérêt (ou non) pour l'approche conceptuelle de l'exposition et le ou les espaces discursifs de l'exposition privilégiés par les enfants dans la relation établie avec l'exposition. À l'aide des trois désignations de la médiation (Gellereau, 2004), à savoir la médiation comme transition, comme intermédiaire et comme lien qui «montrent le rôle de la production de sens» (p. 17), il a été possible de caractériser les récits de médiation des enfants et d'esquisser des figures d'enfants-interprètes dans la perspective de mieux saisir la démarche d'interprétation et de tenter de généraliser.

12. Les sept opérations de la description proposées par Adam et Revaz (1996) nous ont paru adaptées pour rendre compte de ces récits morcelés des enfants. Elles permettent de définir les processus des enfants, plus précisément la manière dont ils dénomment l'objet (ou un ensemble d'objets) par l'opération d'ancrage ou d'affectation, le décrivent en précisant son aspect (opération d'aspectualisation), le mettent en relation selon les dimensions spatiale, temporelle ou par comparaison, et la façon dont ils reformulent leurs propos pour saisir de manière synthétique ce qu'ils ont présenté.

13. Pour une illustration de la démarche d'analyse (cadres d'interprétation et figures d'enfants-interprètes), des exemples d'analyse de «visites guidées» sont données dans les annexes de Martin (2011). 
Ces figures peuvent être déclinées selon différentes approches: la figure de l'explorateur butineur (motivé par sa curiosité, il s'intéresse à tout, même à ce qui peut paraître éloigné du propos de l'exposition); la figure de l'apprenant avide de connaissances, selon l'approche de l'observateur, de l'expérimentateur et du perspicace; la figure du co-constructeur par le partage, selon l'approche du partage de connaissances ou du partage de la sensibilité esthétique; et la figure de l'inventeur passionné, selon l'approche du passionné par son centre d'intérêt, par la scénographie, par le jeu (Martin, 2011). Ces différentes figures sont le reflet de la richesse des expériences de visite (voir le schéma «Des figures d'enfants-interprètes» en annexe).

À propos de la figure de l'apprenant, il est utile de préciser ce qu'il faut entendre par «apprendre». L'espace de médiation s'apparente à «une mise en position d'apprendre ou de goûter» selon Serres (1986; cité dans Caillet, 1995, p. 20). Celui-ci ajoute que cela se rapproche de la conception qu'il se fait "du bon instituteur ${ }^{14}$. L'aventure vers l'apprentissage est rendue possible par l'intermédiaire de la médiation. Il ne s'agit pas ici seulement de s'amuser ou de se distraire pour accéder à la connaissance, mais, plus fondamentalement, de vivre une expérience qui conduit d'un point à un autre, grâce à une médiation participant à l'altérité et constitutive de l'éducation.

Au cours de la visite, la démarche d'appropriation d'un enfant peut revêtir un ou plusieurs aspects. Ces figures qui émergent des paroles et des réactions des enfants sont à distinguer, d'une part, des «catégories de visiteurs" qui enferment dans un mode de fonctionnement et, d'autre part, des figures abstraites ou des modèles qui préétablissent des modes d'appropriation.

\section{L'anthropologie pour accéder à la culture muséale des enfants}

De manière concomitante s'élabore la troisième phase de l'analyse. La méthode ethnographique d'observation in situ et de recueil de paroles d'enfants permet de mettre en lumière les différents mondes qu'ils convoquent pour interpréter et comprendre l'exposition.

Selon Davallon (1986), le monde «utopique» est construit par les visiteurs. Il est défini comme un monde imaginaire. «Il est une construction qui résulte de l'agencement de significations produites au cours des visites, il existe à travers elles» (p. 248) et «il donne lieu chez le visiteur à des activités d'interprétation» (p. 30).

Les enfants font référence aux mondes liés à l'exposition qui regroupent les scientifiques, les objets et leur mise en scène en faisant allusion aux concepteurs. Ils s'appuient aussi sur des références spécifiques à leur propre monde qui se rapportent à leur tissu social, comme l'école et les amis, leurs centres d'intérêt (les dinosaures, le cinéma d'ombres, les fourmis, etc.), et sur leurs expériences antérieures de visite en famille ou, encore, en lien avec d'autres médias. Ce pas qui vient d'être franchi donne accès à la culture muséale des enfants et révèle par la même occasion que la culture se donne à voir par la médiation.

14. «Il y a un moment où il y a une barrière à franchir. C'est l'aventure et son enjeu... Le passage... Pas d'éducation sans expérience de l'altérité. Apprendre, c'est partir. Il faut partir. Tel est le secret absolu de toute éducation. [...] Partir, c'est naître. C'est vivre» (Serres, 1986, cité dans Caillet, 1995, p. 20). 
Ces figures d'enfants-interprètes sont en mesure d'être une source d'inspiration à la fois pour les éducateurs (enseignants, parents), les concepteurs et les médiateurs de l'exposition en vue de considérer la visite des enfants dans un cadre scolaire, selon les dimensions éducative et expérientielle. Le fait de connaître les manières dont les enfants réagissent avec l'environnement et se l'approprient permet aux concepteurs de mettre en œuvre les moyens scénographiques ou aux médiateurs d'identifier une démarche favorisant une expérience de visite qui soit de l'ordre de la rhétorique, ludique ou esthétique, tout en permettant de rattacher ces expériences à des expériences antérieures. Succinctement, cela peut se traduire lors du parcours de visite par des moments qui donnent l'occasion aux jeunes visiteurs de «butiner» et d'explorer tout en s'imprégnant de l'ambiance, d'éprouver du plaisir et d'en témoigner, d'exprimer des points de vue ou des émotions en vue de les partager, de faire part de leurs questions ou de leur compréhension de l'objet (à observer ou à manipuler) ou, encore, de disposer d'un temps pour témoigner de leurs passions, par l'action ou par la parole. La présentation des pistes pour concevoir la visite dans le cadre scolaire a fait l'objet d'une communication à la journée d'études à l'École du Louvre en janvier 2014 (Martin, à paraître).

\section{Des enjeux pour l'accompagnement des enfants et la conception des expositions}

En fonction des modes d'appropriation de l'exposition, il est possible d'envisager plusieurs phases ou «moments» dans la visite afin de permettre l'expression des jeunes visiteurs. Les enfants en général se font une idée du musée et de son rôle. Ce qui détermine aussi leurs façons d'approcher l'exposition. Il est utile de faire émerger ce rôle et les motivations à visiter un musée. Cette préparation à la visite pré-dispose ainsi les visiteurs-enfants à découvrir de manière plurielle l'exposition (en référence aux figures d'enfants-interprètes) favorise un état de réception ouvert prêt à l'exploration d'un monde à explorer ${ }^{15}$; un moment pour découvrir librement: des enfants savent faire preuve de curiosité, se lançant à l'aventure comme des petits détectives avec le sens de l'observation pour percevoir le monde reconstitué par l'exposition; un moment pour faire part de leurs découvertes et pour les partager: lors de la libre exploration, les enfants qui se sont fait une première idée de l'exposition peuvent la partager tout en prenant conscience de la diversité des points de vue sur un même objet ou sur une même salle; un temps pour confronter, éprouver son point de vue: si les interprétations divergent selon la perception des enfants, il peut leur être proposé d'argumenter et de vérifier collectivement ce qui semble le plus pertinent pour comprendre tel phénomène ou événement présenté dans l'exposition. C'est alors que les enfants peuvent préciser sur quoi s'appuient leurs arguments, par exemple sur la lecture des cartels ou leur propre expérimentation.

Des espaces et des dispositifs pour la mise en place de ces moments : la conception des expositions à destination des enfants pourrait prévoir - si ce n'est pas le cas

15. Il peut arriver que des élèves portent leur regard sur un aspect négligé par les adultes mais néanmoins important à leurs yeux. Les accompagnateurs ont tout intérêt à être eux-mêmes attentifs et ouverts aux réactions des enfants. 
- des espaces qui permettent l'expression et les échanges entre enfants, équipés de moyens pour conserver des traces des perceptions ou des hypothèses des enfants qui pourront être complétées et structurées en fonction des raisonnements à l'œuvre. Selon l'organisation de l'exposition, un espace pourrait permettre aux enfants d' "éprouver» par l'observation des objets (ou reproduction d'objets en vitrine) par le toucher, par comparaison tout en exprimant leur sensibilité esthétique (il arrive que ces objets soient déposés dans des malles utilisées lors d'animation dans des musées québécois). L'intérêt de ces espaces est de pouvoir construire collectivement les connaissances grâce à une mise à plat de ce qui a été compris à partir de la perception des enfants, de réajuster leurs représentations si nécessaire par confrontation avec les différents éléments exposés.

\section{Conclusion}

Le fait de rendre accessibles les différentes formes de savoirs du musée à tous les publics, dont les visiteurs-enfants, dans le but de répondre à des objectifs de démocratisation de la culture et du patrimoine laisse entrevoir la nécessité d'une connaissance approfondie des modes d'appropriation des visiteurs-enfants. En nous intéressant, dans notre recherche aux «fabrications culturelles» des enfants pendant la visite, nous avons mis en évidence les potentialités de leur démarche interprétative en fonction de la médiation de l'exposition et avons pu mieux comprendre leurs manières de construire leur «monde utopique», c'est-à-dire ici leurs relations avec le monde de l'exposition.

Cette recherche exploratoire en sciences de l'information et de la communication ouvre de nouveaux horizons pour valoriser le contexte d'éducation non formelle du musée face à une "scolarisation» de la visite et pour cerner ce qui se joue dans l'espace de médiation de l'exposition. Elle contribue à montrer de quelle manière l'expérience globale de visite de l'exposition, dans le cadre des loisirs, participe à la culture muséale des jeunes visiteurs. Elle apporte en même temps un éclairage sur la question de l'acculturation dans les musées de sciences. Ces figures d'enfantsinterprètes aident à mieux appréhender ce jeune public et à comprendre leurs modes d'appropriation au cours de leur expérience de visite. Pour cette raison, cette recherche exploratoire constitue une approche innovante pour réinterroger les façons de s'adresser aux enfants, tant pour les concepteurs que pour les éducateurs. Ce qui participe à l'idée de faire du musée un «outil» de culture, tel que le préconise Davallon (1998). Elle permet de considérer l'éducation muséale sous un angle novateur et d'apporter une contribution aux recherches en muséologie sur les enjeux de la dimension culturelle et éducative de la visite chez les enfants. 


\section{Références bibliographiques}

ADAM, J.-M. et REVAZ, F. (1996). L'analyse des récits. Paris: Seuil.

CAILLET, É. et LEHALLE, É. (1995). À l'approche du musée, la médiation culturelle. Lyon: Presses universitaires de Lyon.

DAVALLON, J. (1986). Claquemurer, pour ainsi dire, tout l'univers. Paris: Centre Georges Pompidou, 241-266.

DAVALLON, J. (1998). Cultiver la science au musée? Dans B. Schiele et H. Koster (dirs.), La révolution de la muséologie des sciences: vers les musées du XXIe siècle (p. 397-434). Lyon: Presses universitaires de Lyon.

DAVALLON, J. (2005). L'exposition à l'œuvre : stratégies de communication et médiation symbolique. Paris: L'Harmattan.

DAVALLON, J., GOTTESDIENER, H. et VILATTE, J.-C. (2006). À quoi peuvent donc servir les recherches sur les visiteurs? Culture et Musées, 8, 161-172.

DE CERTEAU, M. de (1974). La culture aupluriel. Paris: Christian Bourgeois Éditeur.

DE CERTEAU, M. de (2005). L'invention du quotidien. Arts de faire ( $2^{\mathrm{e}}$ éd.). Paris: Folio essais.

DELACÔTE, G. (1996). Savoir apprendre. Les nouvelles méthodes. Paris: Odile Jacob.

DEWEY, J. (2006). Euvres philosophiques III. L'art comme expérience. Pau: Publications de l'Université de Pau, Éditions Farrago.

EIDELMAN, J. et VAN PRAËT, M. (dirs.) (2000). La muséologie des sciences et ses publics. Regards croisés sur la Grande Galerie de l'évolution du Muséum national d'histoire naturelle. Paris: Presses universitaires de France.

GELLEREAU, M. (2004). Construire un monde culturel commun. Pratiques langagières et formes de médiation. Vol. 1 - Parcours de recherche et synthèse des travaux. Habilitation à diriger des recherches en sciences de l'information et de la communication (sous la direction de Bernard Delforce). Villeneuve-d'Ascq: Université Lille 3.

GUICHARD, J. et MARTINAND, J.-L. (2000). Médiatique des sciences. Paris: Presses universitaires de France.

GURIAN, E. H. (2006). Les musées et les jeunes : réflexions à partir de Civilizing the Museum. Nouvelles de l'ICOM, 59(1), 8-13.

JACOBI, D. et COPPEY, O. (1995). Introduction. Musée et éducation : au-delà du consensus, la recherche du partenariat. Publics et Musées, 7, 10-22.

JACOBI, D. et SCHIELE, B. (1990). La vulgarisation scientifique et l'éducation non formelle. Revue française de pédagogie, 91(1), 81-111. 
MARTIN, T. (2011). L'expérience de visite des enfants en musée de sciences dans le cadre des loisirs: les logiques d'interprétation et enjeux d'un dispositif communicationnel (thèse de doctorat en sciences de l'information et de la communication, Université Lille 3, Lille, France). Récupéré le 12 février 2013 de http://tel.archives-ouvertes.fr/tel-00783563

MARTIN, T. (2012). Les logiques d'interprétation des enfants selon leur expérience de visite dans les musées de sciences et dans le cadre des loisirs. Communication, 30(2). Récupéré le 12 février 2013 de http://communication. revues.org/index3598.html

MARTIN, T. (à paraître). Des figures d'enfants-interprètes dans le cadre des loisirs, sources d'inspiration pour la visite scolaire et les modes de transmission. Communication présentée aux Journées d'étude De l'école au musée : quelles conceptions des savoirs, quels modes de transmission? De l'espace scolaire à l'espace muséal, la transmission par l'expérience vécue. Paris: École du Louvre,

MEUNIER, A. (2008). L'éducation muséale, un rapport au savoir. Recherches en communication. 29, 101-124.

MEUNIER, A. (2012). L'éducation muséale, fragment d'une muséologie inachevée? Dans A. Meunier et J. Luckerhoff (dirs.), La muséologie, champ de théories et de pratiques (p. 101-119). Québec: Presses de l'Université du Québec.

PETERS, R. S. (dir.) (2007). John Dewey Reconsidered. Londres: Routledge \& Kegan Paul.

POULOT, D. (2005). Musée et muséologie. Paris: La Découverte.

SCHIELE, B. (2002). Le musée de sciences. Montée du modèle communicationnel et recomposition du champ muséal. Paris: L'Harmattan.

SCREVEN, C. G. (1990). Uses of evaluation before, during and after exhibit design. ILVS Review, 1(2), 36-66.

SILVERSTONE, R. (1998). «Les espaces de la performance : musées, science et rhétorique de l'objet», Hermès, 22, 175-188. 


\section{Annexe}

Des figures d'enfants-interprètes - Thérèse Martin

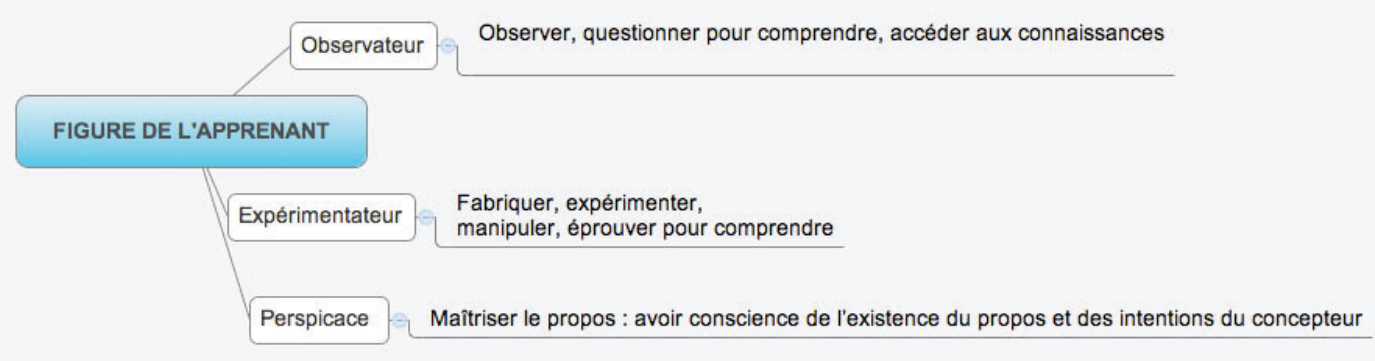

Centre d'intérêt lié à la thématique

Passionné par la scénographie $\odot$ Passion pour la « fabrication » de la scénographie

Passionné par le jeu Plaisir de jouer (manipulations interactives)

Une envie de découvrir, motivé par la curiosité - (sans se centrer pour autant sur le propos de l'exposition) 